\title{
STEADY THERMAL STRESSES IN SOLID DISK UNDER HEAT GENERATION SUBJECTED TO VARIABLE DENSITY
}

\author{
Pankaj Thakur ${ }^{1}$, Satya Bir Singh ${ }^{2},{\text { Joginder } \operatorname{Singh}^{3}, \text { Suresh Kumar }}^{4}$ \\ ${ }^{1}$ Department of Mathematics, IEC University, Baddy, Solan, Himachal Pradesh 174103, India \\ ${ }^{2}$ Department of Mathematics, Punjabi University Patiala, Punjab 147002, India \\ ${ }^{3}$ Department of Mathematics, Chandigarh Engineering College, Landran, Mohali, Punjab \\ ${ }^{4}$ Department of Applied Mathematics, I.K. Gujaral, Punjab Technical University Jalandhar \\ *Corresponding author; E-mail: pankaj_thakur15@yahoo.co.in
}

(Received February 3, 2016)

\begin{abstract}
Seth's transition theory is applied to the problem of stresses in a solid rotating disk under heat generation subjected to variable density by infinitesimal deformation. Neither the yield criterion nor the associated flow rule is assumed here. The results obtained here are applicable to compressible materials. If the additional condition of incompressibility is imposed, then the expression for stresses corresponds to those arising from Tresca yield condition. It has been seen that circumferential stress are maximum at the outer surface for incompressible material as compare to disk made compressible materials. Density variation parameter increases the value of circumferential as well as radial stress at the outer surface of solid disk for compressible and incompressible materials. The present solution is illustrated by numerical results and is compared with heat generation case.
\end{abstract}

Keywords: disk, heat source, stresses, displacement, deformation, speed.

\section{INTRODUCTION}

Rotating disks have a wide range of application in engineering, such as steam and gas turbine, turbo generator, flywheel of internal combustion engines, turbojet engines, reciprocating engines, centrifugal Compressors, brake disk and shrink fit. The analytical elasticity-plasticity of such rotating disks of isotropic materials can be found in many books (TIMOSHENKO et al., 1970, JOHNSON et al., 1978), and the stress analysis in curvilinear orthotropic disks can also found in (LEKHNITSKII et al., 1981) The theoretical and experimental investigations on the rotating solid disk have been widespread attention due to the great practical importance in mechanical engineering. For a better utilization of the material, it is necessary to allow variation of the effective material or thickness properties in one direction of the solid disk. Most of the research works are concentrated on the analytical solutions of rotating isotropic disks with simple cross-section geometries of uniform thickness and specifically variable thickness. The solution of a rotating solid disk with constant thickness is obtained by (GAMER, 1984, 1985) taking into account the linear strain hardening material behavior. (GAMER, 1984) 
found the elastic-plastic deformation of the rotating solid disk under the assumptions of Tresca's yield condition, its associated flow rule and linear strain hardening. To obtain the stress distribution, they matched the plastic stresses at the same radius $r=z$ of the disk. Thermal stresses in a body due to heat sources are encountered in many engineering design and applications. Nuclear reactors are one example; electrical conductors and chemically reacting systems are others.

The thermal stress distribution of a strain hardening sphere due to a uniform heat source was investigated by (DASTIDAR et al., 1972) using the Ramberg-Osgood unaxial stress-strain relation. The effect of temperature on the transverse vibration of a rotating disk of variable thickness was investigated by (GHOSH, 1975) considering a uniform heat source. You et al. (1999) analyzed Elastic-plastic stresses in a rotating solid disk. GUVEN (2000) analyzed elastic-plastic solid disk with non-uniform heat source subjected to external pressure. AHMET et al. (2002) analyzed on the rotating elastic-plastic solid disks of variable thickness having concave profiles. THAKUR et al. (2015) investigated Infinitesimal deformation in a solid Disk by using Seth's transition theory. THAKUR et al. (2015) studied elastic-plastic infinitesimal deformation in a solid disk under heat effect by using Seth's theory. THAKUR et al. (2015) investigated thermo elastic-plastic deformation in a solid disk with heat generation subjected to pressure.

\section{METHODOLOGY}

(SETH, 1962) proposed transition Theory and defined generalized strain measure. Seth's defined transition function for elastic-plastic and creep state. When a deformable solid is subjected to an external loading system, it has been observed that the solid first deforms elastically. If the loading is continued, plastic flow may set in, and if continued further, it gives rise to time dependent continuous deformation known as creep deformation. It may be possible that a number of transition states may occur at the same critical point, then the transition function will have different values, and the point will be a multiple point, each branch of which will then correspond to a different state. In general, the material from elastic state can go over into (i) plastic state, or to (ii) creep state, or (iii) first to plastic state and then to creep and vice-versa, depending upon the loading.

All these final states are reached through a transition state. In the plane stress condition, the transition can take place either through the principal stresses $\tau_{r r}$ or $\tau_{\theta \theta}$ becoming critical or through the principal stress difference $\tau_{r r}-\tau_{\theta \theta}$ becoming critical. Hence we have to consider the following three cases: (a) Transition through $\tau_{r r}$, (b) Transition through $\tau_{\theta \theta},(c)$ Transition through $\tau_{r r}-\tau_{\theta \theta}$. For each transition point, we have to determine the stresses and strains corresponding to the above three cases. Seth's transition theory does not acquire any assumptions like a yield condition, incompressibility condition and thus poses and solves a more general problem from which cases pertaining to the above assumptions can be worked out. It utilizes the concept of generalized strain measure and asymptotic solution at critical points or turning points of the differential equations defining the deforming field and has been successfully applied to a large number of the problems (GUPTA et al., 2007, 2008) and (THAKUR et al., 2010, 2014, 2015). Results have been discussed numerically and depicted graphically. In general, steady-state temperature heat generation may be a function of space or temperature (BAYAZITOGLU et al., 2008). This work is concerned with the steady thermal stresses in a solid disk under heat generation subjected to variable density. For the problem considered here, heat generation rate $\dot{q}(r)$ is a function of the radial position in the form 
$\dot{q}(r)=q_{0}\left[1-\left(\frac{r}{a}\right)^{s}\right]$

where $q_{0}$ is the magnitude of the heat generation at $r=0, r$ is measured from the centre of solid disk, $a$ is the radius of disk. The analysis is based on the usual assumptions of a plane stress state. The density of the disk vary along the radius in the form

$\rho=\rho_{0}(r / a)^{-m}$

where $\rho_{0}$ is the constant density at $r=a$ and $m$ is the density parameter

\section{MATHEMATICAL MODEL AND BASIC EQUATIONS OF THE PROBLEM)}

We consider a state of plane stress and assume infinitesimal deformation. Suppose that solid disk having of variable density with radius $a$. The disk is rotating with angular velocity $\omega$ about an axis perpendicular to its plane and passing through the centre. The thickness of disk is assumed to be constant and is taken to be sufficiently small so that the disk is effectively in a state of plane stress that is, the axial stress $T_{z z}$ is zero. The cylindrical polar coordinates are given by (SETH, 1962):

$u=r(1-\beta) ; v=0 ; w=d z$

where $\beta$ is function of $r=\sqrt{x^{2}+y^{2}}$ only and $d$ is a constant.

The strain components for infinitesimal deformation are:

$\stackrel{A}{\mathcal{E}}_{r r} \equiv \frac{\partial u}{\partial r}=\left[1-\left(r \beta^{\prime}+\beta\right)\right], \stackrel{A}{\mathcal{E}_{\theta \theta}} \equiv \frac{u}{r}=[1-\beta], \quad \stackrel{A}{\mathcal{E}_{z z}} \equiv \frac{\partial w}{\partial z}=d, \quad \stackrel{A}{\mathcal{E}_{r \theta}}=\stackrel{A}{\mathcal{E}_{\theta z}}=\stackrel{A}{\mathcal{E}_{z r}}=0$

The generalized components of strain are given (THAKUR, 2010):

$\mathcal{E}_{r r}=\frac{1}{n}\left[1-\left\{2\left(r \beta^{\prime}+\beta\right)-1\right\}^{n / 2}\right], \varepsilon_{\theta \theta}=\frac{1}{n}\left[1-\{2 \beta-1\}^{n / 2}\right], \varepsilon_{z z}=\frac{1}{n}\left[1-(1-2 d)^{n / 2}\right], \varepsilon_{r \theta}=\varepsilon_{r \theta}=\varepsilon_{z r}=0$

where $\beta^{\prime}=d \beta / d r$. The stress - strain relations for isotropic media is given by (Sokolnikoff, 1952):

$\tau_{i j}=\lambda \delta_{i j} I_{1}+2 \mu \varepsilon_{i j}-\xi \Theta \delta_{i j},(i, j=1,2,3)$

where $\tau_{i j}$ is the stress components $\varepsilon_{i j}$ strain components $\lambda, \mu$ are Lame's constants $I_{1}=\varepsilon_{k k}$ is the first strain invariant, $\delta_{i j}$ is the Kronecker's delta where $\xi=\alpha(3 \lambda+2 \mu) ; \alpha$ being the coefficient of thermal expansion and $\Theta$ is a temperature. The mathematical formulation of the steady-state one-dimensional heat flow with heat source is given (BAYAZITOGLU et al., 2008) by

$$
\frac{1}{r} \frac{d}{d r}\left[r \frac{d \Theta(r)}{d r}\right]+\frac{\dot{q}(r)}{k}=0 \quad \text { in } 0<r<a
$$

The temperature field satisfying equation (6) and $\frac{d \Theta(r)}{d r}=0$ at $r=0, \Theta(r)=0$ at $r=a$. Using equation 1(a) and these boundary conditions, the steady-state temperature distribution is obtained as: 
$\Theta(r)=\frac{q_{0} a^{2}}{4 k}\left[1-\left(\frac{r}{a}\right)^{2}-\frac{4}{(s+2)^{2}}\left[1-\left(\frac{r}{a}\right)^{s+2}\right]\right] ;$ where $k$ is the thermal conductivity. Equation (5) for this problem becomes:

$\tau_{r r}=\frac{2 \lambda \mu}{\lambda+2 \mu}\left[\varepsilon_{r r}+\varepsilon_{\theta \theta}\right]+2 \mu \varepsilon_{r r}-\frac{2 \mu \xi \Theta}{\lambda+2 \mu}, \tau_{\theta \theta}=\frac{2 \lambda \mu}{\lambda+2 \mu}\left[\varepsilon_{r r}+\varepsilon_{\theta \theta}\right]+2 \mu \varepsilon_{\theta \theta}-\frac{2 \mu \xi \Theta}{\lambda+2 \mu}$,

$\tau_{z z}=\tau_{z r}=\tau_{r \theta}=\tau_{\theta z}=0$.

From equation (5), strain components in terms of stresses are obtained as:

$\varepsilon_{r r}=\frac{\partial u}{\partial r}=\frac{1}{E}\left[\tau_{r r}-v \tau_{\theta \theta}\right]+\alpha \Theta, \varepsilon_{\theta \theta}=\frac{u}{r}=\frac{1}{E}\left[\tau_{\theta \theta}-v \tau_{r r}\right]+\alpha \Theta, \varepsilon_{z z}=-\frac{v}{E}\left[\tau_{r r}-\tau_{\theta \theta}\right]+\alpha \Theta, \varepsilon_{r r}=\varepsilon_{\theta z}=\varepsilon_{z r}=0$

where $E=\frac{\mu(3 \lambda+2 \mu)}{(\lambda+\mu)}$ and $v=\frac{\lambda}{2(\lambda+\mu)}$. Substituting equation (4) in equation (7), the stresses are obtained as:

$\tau_{r r}=\frac{2 \mu}{n}\left[3-2 c-\{2 \beta(P+1)-1\}^{n / 2}(2-c)-(2 \beta-1)^{n / 2}(1-c)-\frac{n c \xi \Theta}{2 \mu}\right]$,

$\tau_{\theta \theta}=\frac{2 \mu}{n}\left[3-2 c-\{2 \beta(P+1)-1\}^{n / 2}(1-c)-(2 \beta-1)^{n / 2}(2-c)-\frac{n c \xi \Theta}{2 \mu}\right], \tau_{z z}=\tau_{z r}=\tau_{r \theta}=\tau_{\theta z}=0$,

where $r \beta^{\prime}=\beta P$ and $c=\frac{2 \mu}{\lambda+2 \mu}$. The equations of equilibrium are all satisfied except:

$\frac{d}{d r}\left(r \tau_{r r}\right)-\tau_{\theta \theta}+\rho \omega^{2} r^{2}=0$

Using equations (9) and (6) in equation (10), we get a non -linear differential equation in $\beta$ as:

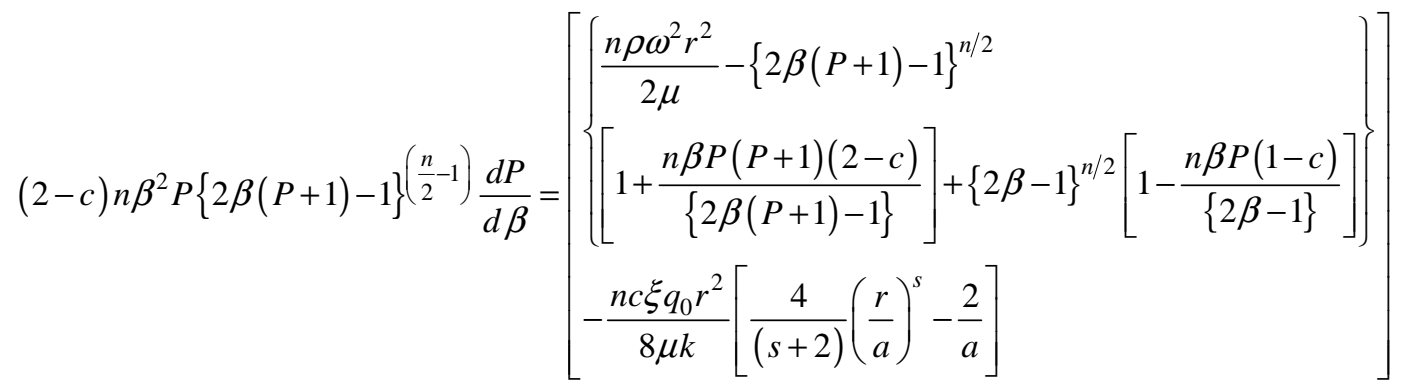

where $P\left(P\right.$ is function of $\beta$ and $\beta$ function of $\mathrm{r}$ )and $\Theta^{\prime}(r)=\frac{q_{0} a^{2}}{4 k}\left[\frac{4}{(s+2)}\left(\frac{r}{a}\right)^{s+1}-\frac{2}{a}\right]$.

TRANSITION POINTS: Transition points of $\beta$ in equation (11) are $P \rightarrow 0$ and $P \rightarrow \pm \infty . P \rightarrow 0$ gives nothing of importance.

BOUNDARY CONDITION: The solid disk considered in the present study is with heat generation. The inner surface of the disk is assumed to be zero. The outer surface of the disk is applied mechanical load. Thus, the boundary conditions of the problem are given by:

$u=0 \quad$ at $\quad r=0$ and $\tau_{r r}=0$ at $r=a$ (12)

\section{SOLUTION OF THE PROBLEM}

For finding the plastic stress, the transition function is taken through the principal stress (see Seth, 1962, 1966; Gupta et al., 2007, 2008; Thakur et al., 2010, 2014, 2015) at the transition point $P \rightarrow \pm \infty$. We define the transition function $R$ as: 
$\varsigma=\frac{n}{2 \mu}\left[\tau_{\theta \theta}+C \xi \Theta\right]=\left[(3-2 c)-\{2 \beta(P+1)-1\}^{n / 2}(1-c)-(2 \beta-1)^{n / 2}(2-c)\right]$

where $\varsigma$ transition function of $r$.

Taking the logarithmic differentiation of equation (13) with respect to $r$ and using equation (11), we get:

$\frac{d(\log \varsigma)}{d r}=-\frac{\left(\frac{1-c}{2-c}\right)\left[\begin{array}{l}\frac{n \rho \omega^{2} r^{2}}{2 \mu \beta^{n}}-\{2 \beta(P+1)\}^{n / 2}+(2 \beta-1)^{n / 2} \\ -n \beta P(1-c)(2 \beta-1)^{n / 2} \\ -\frac{n c \xi(d \Theta / d r)}{2 \mu \beta^{n}}\end{array}\right]+n \beta P(2-c)(2 \beta-1)^{n / 2}}{r\left[\begin{array}{l}3-2 c-\{2 \beta(P+1)-1\}^{n / 2}(1-c) \\ -(2 \beta-1)^{n / 2}(2-c)\end{array}\right]}$

Taking the asymptotic value of equation (14) as $P \rightarrow \pm \infty$ and after integration we get:

$\varsigma=\operatorname{Ar}^{-1 /(2-c)}$

where $A$ is a constant of integration which can be determined by boundary condition. From equation (13) and (15), we have:

$\tau_{\theta \theta}=\left\{\left(\frac{2 \mu}{n}\right) \operatorname{Ar}^{-1 /(2-C)}-\frac{c \xi q_{0} a^{2}}{4 k}\left[1-\left(\frac{r}{a}\right)^{2}-\frac{4}{(s+2)^{2}}\left[1-\left(\frac{r}{a}\right)^{s+2}\right]\right]\right\}$

Substituting (16) in equation (10) and using 1(b) after integrating, we get:

$\tau_{r r}=\left\{\frac{2 \mu(2-c)}{n(1-c)}\right\} A r^{-1 /(2-C)}-\frac{c \xi q_{0} a^{2}}{4 k}\left[\begin{array}{l}1-\left(\frac{r}{a}\right)^{2} \\ -\frac{4}{(s+2)^{2}}\left[1-\left(\frac{1}{s+3}\right)\left(\frac{r}{a}\right)^{s+2}\right]\end{array}\right]-\frac{\rho_{0} \omega^{2} r^{2-m}}{(3-m) b^{-m}}+\frac{B}{r}$

where $B$ is a constant of integration which can be determined by boundary condition. Substituting equations (16) and (17) in equation (8), we get:

$$
\begin{aligned}
& \frac{\partial u}{\partial r}=\frac{1}{E}\left[\left(\frac{2 \mu}{n}\right) A r^{-1 / 2-C}\left\{\frac{3-2 c}{(1-c)(2-c)}\right\}+\frac{\alpha E q_{0} a^{2}(2-c)}{k(s+2)(s+3)}\left(\frac{r}{a}\right)^{s+2}-\frac{\rho_{0} \omega^{2} r^{2-m}}{(3-m) b^{-m}}+\frac{B}{r}\right] \\
& \frac{u}{r}=\frac{(1-c)}{E(2-c)}\left[\frac{\rho_{0} \omega^{2} r^{2-m}}{(3-m) b^{-m}}-\frac{\alpha E q_{0} a^{2}(1-c)}{2 k}\left[-1+\left(\frac{r}{a}\right)^{2}-\frac{2(s+4)}{(s+2)^{2}(s+3)}\left(\frac{r}{a}\right)^{s+2}\right]-\frac{B}{r}\right]
\end{aligned}
$$

where $E=\frac{2 \mu(3-2 c)}{(2-c)}$ is the Young's modulus. Integrating equation (18) with respect to $r$, we gets:

$u=\frac{1}{E}\left[\left(\frac{2 \mu}{n}\right) A_{1} r^{\frac{1-c}{2-c}}\left\{\frac{(3-2 c)}{(1-c)^{2}}\right\}+\frac{\alpha E q_{0} a^{2}(2-c)}{k(s+2)(s+3)^{2}}\left(\frac{r}{a}\right)^{s+3}-\frac{\rho_{0} \omega^{2} r^{3-m}}{(3-m)^{2} b^{-m}}+B_{1} \log r\right]+D$

where $D$ is a constant of integration which can be determined by boundary condition. Comparing equations (19) and (20), one gets: 
$\left(\frac{2 \mu}{n}\right) A_{1} r^{\frac{1-c}{2-c}}\left\{\frac{3-2 c}{(1-C)^{2}}\right\}=\left[\frac{\rho \omega^{2} r^{3-m}}{(3-m) b^{-m}}\left(\frac{1-c}{2-c}+\frac{1}{3-m}\right)-\frac{\alpha E q_{0} a^{2}}{k}\left[\begin{array}{l}\frac{(2-c) a}{(s+2)(s+3)^{2}}\left(\frac{r}{a}\right)^{s+3}+ \\ \frac{r(1-c)^{2}}{2(2-c)}\left\{\begin{array}{l}\left(\frac{r}{a}\right)^{2}-1 \\ -\frac{2(s+4)}{(s+2)^{2}(s+3)}\left(\frac{r}{a}\right)^{s+2}\end{array}\right]\end{array}\right]-B_{1}\left\{\frac{(1-c)+(2-c) \log r}{(2-c)}\right\}-D E\right]$

and $u=\frac{(1-c)}{E(2-c)}\left[\frac{\rho \omega^{2} r^{3-m}}{(3-m) b^{m}}-\frac{\alpha E q_{0} a^{2}(1-c) r}{2 k}\left\{-1+\left(\frac{r}{a}\right)^{2}-\frac{2(s+4)}{(s+2)^{2}(s+3)}\left(\frac{r}{a}\right)^{s+2}\right\}\right]$

Using boundary conditions (12) in equations (22), we gets: $B=0$. Putting equations (21) in equation (17) and using boundary condition (11), we get:

$D=\frac{1}{E}\left[\frac{\rho_{0} \omega^{2} a^{3}}{(3-m)}\left(\frac{1-c}{2-c}+\frac{1}{3-m}-\frac{3-2 c}{(1-c)(2-c)}\right)-\frac{\alpha E q_{0} a^{3}}{k(s+2)(s+3)}\left\{\left(\frac{2-c}{s+3}\right)-\frac{(1-c)^{2}(s+4)}{(2-c)(s+2)}+\frac{3-2 c}{1-c}\right\}\right]$ Putting values of $B_{1}, D$ and using equations (21) in equations (16) and (17) respectively, we get the plastic stresses and displacement as:

$$
\begin{aligned}
& {\left[\frac{\rho_{0} \omega^{2}}{(3-m)}\left(\frac{r^{3-m}-a^{3-m}}{a^{-m}}\right)\left\{\frac{(1-c)(2-c)}{(3-2 c)}\left(\frac{1-c}{2-c}+\frac{1}{3-m}\right)-1\right\}-\frac{\alpha E q_{0} a^{3}(2-c)^{2}}{k(s+2)(s+3)^{2}(3-2 c)}\left\{\left(\frac{r}{a}\right)^{s+3}-1\right\}\right.} \\
& r \tau_{r r}=-\frac{\alpha E q_{0} a^{2}(1-c)^{3} r}{2 k(3-2 c)}\left\{\left(\frac{r}{a}\right)^{2}-1\right\}+\frac{\alpha E q_{0} a^{3}(2-c)(1-c)^{3}(s+4)}{k(s+2)^{2}(s+3)(3-2 c)}\left\{\left(\frac{r}{a}\right)^{s+3}-1\right\}-\frac{\alpha E q_{0} a^{2}(2-c) r}{4 k}\left\{1-\left(\frac{r}{a}\right)^{2}\right\} \\
& +\frac{\alpha E q_{0} a^{3}(2-c)}{k(s+2)(s+3)}\left\{1-\left(\frac{r}{a}\right) \frac{(s+3)}{s+2}+\frac{1}{(s+2)}\left(\frac{r}{a}\right)^{s+3}\right\} \\
& {\left[\frac{\rho_{0} \omega^{2}(1-c)^{2}}{(3-m)(3-2 c) a^{-m}}\left[\left(r^{3-m}-a^{3-m}\right)\left(\frac{1-c}{2-c}+\frac{1}{3-m}\right)+a^{3-m}\left\{\frac{3-2 c}{(1-c)(2-c)}\right\}\right]\right.} \\
& r \tau_{\theta \theta}=\left\{-\frac{\alpha E q_{0} a^{3}(1-c)(2-c)}{k(s+2)(s+3)^{2}(3-2 c)}\left\{\left(\frac{r}{a}\right)^{s+3}-1\right\}-\frac{\alpha E q_{0} a^{2}(1-c)^{4} r}{2 k(3-2 c)(2-c)}\left\{\left(\frac{r}{a}\right)^{2}-1\right\}\right. \\
& {\left[+\frac{\alpha E q_{0} a^{3}(1-c)^{4}(s+4)}{k(s+2)^{2}(s+3)(2-c)(3-2 c)}\left\{\left(\frac{r}{a}\right)^{s+2}-1\right\}-\frac{\alpha E q_{0} a^{2}(2-c) r}{k(s+2)^{2}}\left\{1-\left(\frac{r}{a}\right)^{2}\right\}+\frac{\alpha E q_{0} a^{3}(1-c)}{k(s+2)(s+3)}\right]} \\
& u=\frac{(1-c)}{E(2-c)}\left[\frac{\rho \omega^{2} r^{3-m}}{(3-m) b^{m}}-\frac{\alpha E q_{0} a^{2}(1-c) r}{2 k}\left\{-1+\left(\frac{r}{a}\right)^{2}-\frac{2(s+4)}{(s+2)^{2}(s+3)}\left(\frac{r}{a}\right)^{s+2}\right\}\right]
\end{aligned}
$$

Initial Yielding: For a solid disk the stress at the centre is given when $r=0$. With $r$ equal to zero the above equations will yield infinite stresses whatever the speed of rotation, these stresses are not meaningful. Suppose we take yielding at $r=r_{1}$ (say), then equation (23) becomes: 


$$
\left|r \tau_{r r}\right|_{r=r_{1}}=\left[\begin{array}{l}
\frac{\rho_{0} \omega^{2}}{(3-m)}\left(\frac{r_{1}^{3-m}-a^{3-m}}{a^{-m}}\right)\left\{\frac{(1-c)(2-c)}{(3-2 c)}\left(\frac{1-c}{2-c}+\frac{1}{3-m}\right)-1\right\}-\frac{\alpha E q_{0} a^{3}(2-c)^{2}}{k(s+2)(s+3)^{2}(3-2 c)}\left\{\left(\frac{r_{1}}{a}\right)^{s+3}-1\right\} \\
-\frac{\alpha E q_{0} a^{2}(1-c)^{3} r_{1}}{2 k(3-2 c)}\left\{\left(\frac{r_{1}}{a}\right)^{2}-1\right\}+\frac{\alpha E q_{0} a^{3}(2-c)(1-c)^{2}(s+4)}{k(s+2)^{2}(s+3)(3-2 c)}\left\{\left(\frac{r_{1}}{a}\right)^{s+3}-1\right\}-\frac{\alpha E q_{0} a^{2}(2-c) r_{1}}{4 k}\left\{1-\left(\frac{r_{1}}{a}\right)^{2}\right\} \\
+\frac{\alpha E q_{0} a^{3}(2-c)}{k(s+2)(s+3)}\left\{-\left(\frac{r_{1}}{a}\right) \frac{(s+3)}{s+2}+\frac{1}{(s+2)}\left(\frac{r_{1}}{a}\right)^{s+3}\right\}
\end{array}\right]
$$

where $\sigma_{0}$ denotes the initial yield stress. The angular speed necessary for inner-plastic-zone is given by:

$$
\Omega^{2}=\bmod \frac{\left[\begin{array}{l}
\frac{Q_{1}(2-c)^{2}\left(x_{1}^{s+3}-1\right)}{(s+2)(s+3)^{2}(3-2 c)}+\frac{Q_{1}(1-c)^{3}\left(x_{1}^{2}-1\right)}{2(3-2 c)}-\frac{Q_{1}(2-c)(1-c)^{2}(s+4)\left(x_{1}^{s+3}-1\right)}{(s+2)^{2}(s+3)(3-2 c) x_{1}}+\frac{Q_{1}(2-c)\left(1-x_{1}^{2}\right)}{4} \\
-\frac{Q_{1}(2-c)}{(s+2)(s+3) x_{1}}\left\{1-x_{1}\left(\frac{s+3}{s+2}\right)+\frac{1}{s+2} x_{1}^{s+3}\right\}
\end{array}\right]}{\frac{1}{(3-m) r_{1}}\left(x_{1}^{3-m}-1\right)\left\{\frac{(1-c)(2-c)}{(3-2 c)}\left(\frac{1-c}{2-c}+\frac{1}{3-m}\right)-1\right\}}
$$

where $x_{1}=\left(0<r<r_{1}\right) / a(=0.4), Q=\frac{E \alpha q_{0} a^{2}}{k \sigma_{0}} ; \sigma_{r}=\frac{\tau_{r r}}{\sigma_{0}}$. The stresses and displacement are obtained in non dimension form as:

$$
\begin{aligned}
& \sigma_{r}=\left[\begin{array}{l}
\frac{\Omega^{2}}{(3-m) x_{1}}\left(x_{1}^{3-m}-1\right)\left\{\frac{(1-c)(2-c)}{(3-2 c)}\left(\frac{1-c}{2-c}+\frac{1}{3-m}\right)-1\right\}-\frac{Q_{1}(2-c)^{2}\left(x_{1}^{s+3}-1\right)}{(s+2)(s+3)^{2}(3-2 c) x_{1}}-\frac{Q_{1}(1-c)^{3}\left(x_{1}^{2}-1\right)}{2(3-2 C)} \\
+\frac{Q_{1}(2-c)(1-c)^{2}(s+4)\left(x_{1}^{s+3}-1\right)}{(s+2)^{2}(s+3)(3-2 C) x_{1}}-\frac{Q_{1}(2-c)\left(1-x_{1}^{2}\right)}{4}+\frac{Q_{1}(2-c)}{(s+2)(s+3) x_{1}}\left\{1-x_{1} \frac{(s+3)}{s+2}+\frac{1}{(s+2)} x_{1}^{s+3}\right\}
\end{array}\right] \\
& \sigma_{\theta}=\left[\begin{array}{l}
\frac{\Omega^{2}(1-c)^{2}(5-4 c)}{(3-m) x_{1}(3-2 c)}\left[\begin{array}{l}
\left(x_{1}^{3}-1\right)\left\{\frac{1-c}{2-c}+\frac{1}{3-m}\right\} \\
+\frac{3-2 c}{(1-c)(2-c)}
\end{array}\right]-\frac{Q_{1}(1-c)(2-c)\left(x_{1}^{s+3}-1\right)}{(s+2)(s+3)^{2}(3-2 c) x_{1}}-\frac{Q_{1}(1-c)^{4}\left(x_{1}^{2}-1\right)}{2(3-2 c)(2-c)} \\
+\frac{Q_{1}(1-c)^{4}(s+4)\left(x_{1}^{s+2}-1\right)}{(s+2)^{2}(s+3)(2-c)(3-2 C) x_{1}}-\frac{Q_{1}(2-c)\left(1-x_{1}^{2}\right)}{(s+2)^{2}}+\frac{Q_{1}(1-c)}{(s+2)(s+3) x_{1}}
\end{array}\right] \\
& \bar{u}=\frac{(1-c)}{(2-c)\left[\frac{\Omega_{i}^{2}}{(3-m)} x_{1}^{3-m}-\frac{Q_{1}(1-c) x_{1}}{2}\left\{-1+x_{1}^{2}-\frac{2(s+4)}{(s+2)^{2}(s+3)} x_{1}^{s+2}\right\}\right]}
\end{aligned}
$$

where $\sigma_{\theta}=\frac{\tau_{\theta \theta}}{\sigma_{0}} ; u=\frac{\bar{u} \sigma_{0} a}{E}$.

\section{RESULTS AND DISCUSSION}

To see the effect of stresses and displacement distribution in a rotating solid disk following values have been taken $c=0$ (Incompressible material), 0.25 (Compressible material), 0.75 (Compressible material), $Q_{1}=200,400$ and $s=3$. In Figure 2-3, curve has been drawn between stresses and displacement of the rotating solid disk having density parameter $(m=0$, 0.25 . 0.5) and having different values of heat source along the radii ratio $x_{1}(r / a)$. It has been seen that circumferential stress are maximum at the outer surface for incompressible material 
as compare to disk made compressible materials. Density variation parameter increases the value of circumferential as well as radial stress at the outer surface of solid disk for compressible and incompressible materials. With effect of heat generation, stresses and the displacement must be increase for compressible and incompressible materials.

\section{CONCLUSION}

It has been seen that circumferential stress are maximum at the outer surface for incompressible material as compare to disk made compressible materials. Density variation parameter increases the value of circumferential as well as radial stress at the outer surface of solid disk for compressible and incompressible materials.

\section{Acknowledgment}

The authors gratefully acknowledge UGC, New Delhi for providing financial support to carry out this research work under UGC-Major Research Project Scheme (MRP-MAJORMATH-2013-41603).

\section{References:}

[1] Bayazitoglu, Y., Ozisik, N., Elements of heat transfer. New York: McGraw-Hill (1988).

[2] Dastidar, D.G., Ghosh, P., Temperature stress distribution in a sphere due to uniform heat source for strain hardening material. Journal of Applied Mechanics 39 (1972) 274276.

[3] ERASLAN, A.N., ORCAN, YUSUF, Elastic-plastic deformation of a rotating solid disk of exponentially varying thickness, Mechanics of Materials 34 (7) (2002) 423-432 DOI: 10.1016/S0167-6636(02)00117-5.

[4] GAMER, U., Elastic-plastic deformation of the rotating solid disk. Ingenieur-Archiv 54 (1984) 345-354.

[5] GAMER, U., Stress distribution in the rotating elastic-plastic disk. ZAMM 65 (1985) T136-137.

[6] GHOSH, N.C., Thermal effect on the transverse vibration of spinning disk of variable thickness. Journal of Applied Mechanics 42 (1975) 358-362.

[7] Gupta, S.K., PANKaJ ThakuR, Creep Transition in a thin rotating disc with rigid Inclusion, Defence Science Journal, India 57 (2) (2007) 185-195.

[8] Gupta, S.K., PAnKaJ Thakur, Thermo Elastic-plastic transition in a thin rotating Disc with inclusion, Thermal Science 11 (1) (2007) 103-118.

[9] Gupta, S.K., PAnKaJ ThakuR, Creep Transition in an isotropic disc having variable thickness subjected to internal pressure, Proceeding National Academy of Science, India, Springer, Sec.-A, 78 (1) (2008) 57-66.

[10] Guven, U., Altay, O., Elastic-plastic solid disk with non uniform heat source subjected to external pressure, International Journal of Mechanical Sciences 42 (5) (2000) 831-842. 
[11] Johnson, W., Mellor, P.B., Engineering plasticity, Von-Nostrand Reinhold Company, Londan (1978).

[12] LEKHNITSKII S.G., Theory of elasticity of an anisotropic body, Moscow, Mir (1981) 6678.

[13] PAnKaj Thakur, Satya BIR Singh, Infinitesimal deformation in a solid Disk by using Seth's transition theory, International Journal of Electro-mechanics and Mechanical Behavior 1 (1) (2015) 1-6.

[14] Pankaj Thakur, Satya Bir Singh, Shruti Sawhney, Elastic-plastic infinitesimal deformation in a solid disk under heat effect by using Seth Theory, International Journal of Applied and Computational Mathematics, online 13 Nov. 2015, 13pp. DOI:10.1007/s40819-015-0116-9.

[15] Pankaj ThakUR, Satya Bir Singh, Jasmina Lozanović Šajić, Thermo elastic-plastic deformation in a solid disk with heat Generation subjected to pressure, Structure Integrity and Life 15 (3) (2015) 135-142.

[16] PANKaj Thakur, Elastic-plastic transition stresses in a thin rotating disc with rigid inclusion by infinitesimal deformation under steady state Temperature. Thermal Science 14 (1) (2010) 209-219.

[17] PANKaJ ThakuR, Steady thermal stress and strain rates in a rotating circular cylinder under steady state temperature, Thermal Science 18 (suppl. 1) (2014) 93-106.

[18] Pankaj Thakur, Jatinder Kaur, SAtya Bir Singh, Thermal creep transition stresses and strain rates in a circular Disc with shaft having variable density, Engineering Computations, Emerald Publisher UK, 33 (2) (2015) 698-712. DOI: http://dx.doi.org/10.1108/EC-05-2015-0110

[19] Pankaj Thakur, Satya Bir Singh, Suresh Kumar, Mechanical Load in a circular rotating Disk With Shaft For Different Materials Under Steady-State Temperature, Scientific Technical Review, Military Technical Institute Ratka Resanovića, Belgrade, Serbia 65 (1) (2015) 36-42.

[20] Pankaj Thakur, Satya BiR Singh, Jatinder KaUR, Elastic-plastic stresses in a thin rotating disk with shaft having density variation parameter under steady-state temperature. Kragujevac Journal of Science 36 (2014) 5-17.

[21] PANKAJ ThaKUR, SATYA BIR SINGH, Elastic-plastic transitional stresses distribution and displacement for transversely isotropic circular disc with inclusion subject to mechanical load, Kragujevac Journal of Science 37 (2015) 25-36.

[22] SeTH B.R., Transition theory of Elastic- plastic deformation, Creep and relaxation, Nature, 195 (1962) 896 -897.

[23] Seth B.R., Measure concept in Mechanics, Int. J. Non-linear Mech. 1 (1966) 35- 40.

[24] SoKolnikoff, I.S., Mathematical theory of Elasticity, $2^{\text {nd }}$ edition, New York, (1952) pp. 65-79.

[25] Timoshenko, S., GoodiEr, J.N., Theory of Elasticity, McGraw-Hill, New York (1970).

[26] You, L.H., ZHANG, J.J., Elastic-plastic stresses in a rotating solid disk, International Journal of Mechanical Sciences 41 (3) (1999) 269-282. 


$$
Q_{1}(\text { Heat generation })=200
$$
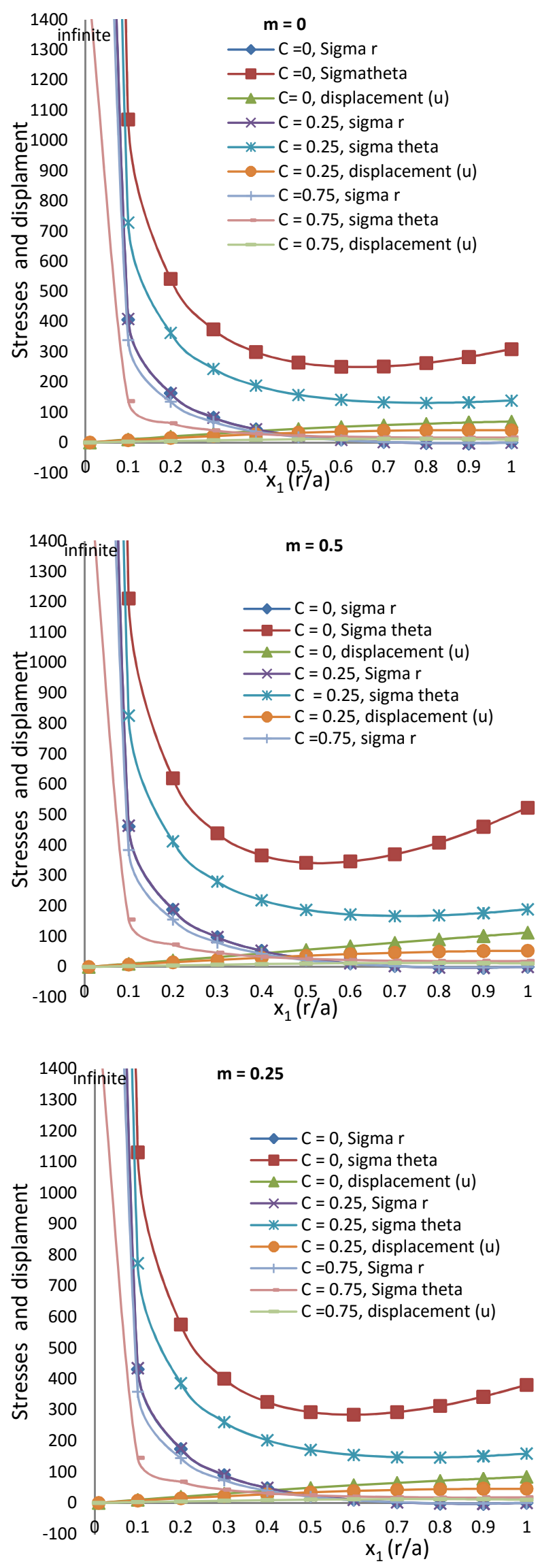

Figure 2. Stresses and displacement distribution in solid disk.
$Q_{1}($ Heat generation $)=400$
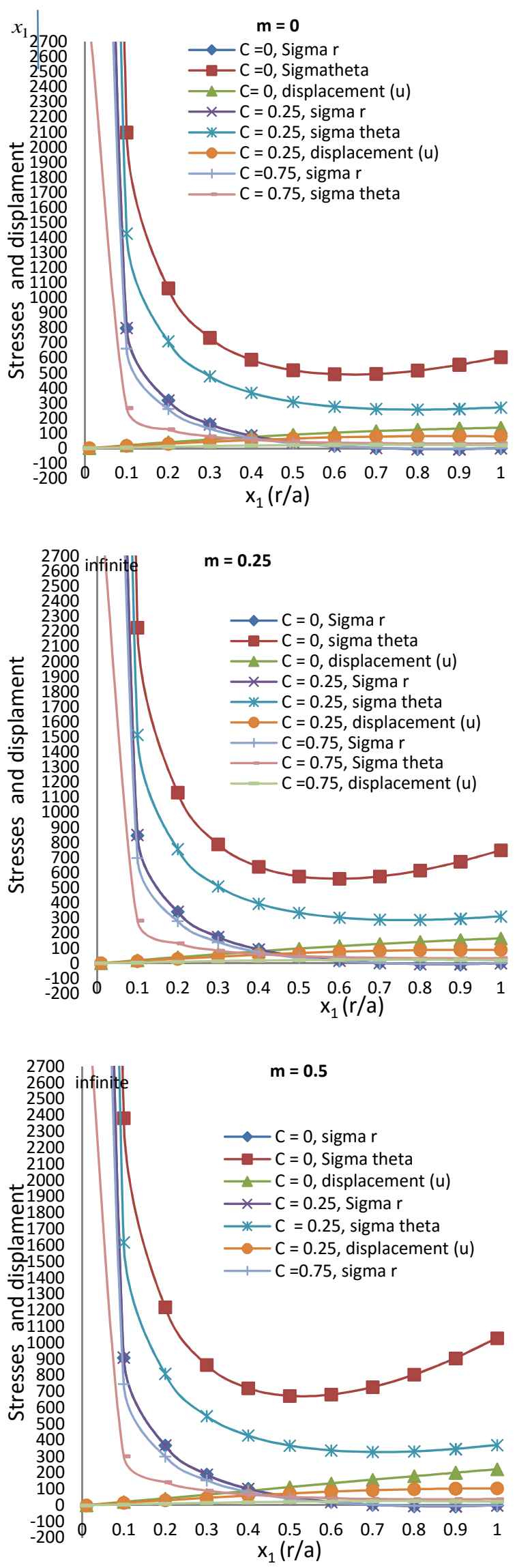

Figure 3. Stresses and displacement distribution in solid disk. 